\title{
Distribución espacial de la macrofauna en una playa expuesta de Chile central: efectos de la morfodinámica intermareal
}

\author{
Alejandro Brazeiro, Nicolás Rozbaczylo y José Miguel Fariña \\ Departamento de Ecología, Facultad de Ciencias Biológicas \\ Pontificia Universidad Católica de Chile, Casilla 114-d, Santiago, Chile \\ E-mail: eabrazei@genes.bio.puc.cl
}

\begin{abstract}
RESUMEN. Si bien la heterogeneidad espacial en la abundancia de organismos (parchosidad) es un fenómeno recurrente en playas de arena, ha recibido escasa atención por parte de los ecólogos. Existe una amplia documentación de la ocurrencia de parchosidad en poblaciones de playas de arena, pero en general se desconocen los factores causales de la misma. En este trabajo proponemos que la variabilidad espacial de la morfodinámica intermareal, afecta a la densidad y distribución espacial (zonación) de la macrofauna a lo largo de la playa. En tal sentido, analizamos el efecto de las variaciones espaciales en la morfodinámica intermareal sobre la zonación y densidad de la macrofauna de una playa de Chile central, en base a la comparación entre sitios de morfología contrastante: valles y cúspides. Comparativamente, los valles fueron sectores de menor pendiente, mayor amplitud intermareal, menor frecuencia de barrido, y presentaron un menor porcentaje de arena gruesa. En general las especies de la macrofauna tendieron a ser más abundantes en los valles, sin embargo Excirolana braziliensis fue la única especie que presentó diferencias significativas. De acuerdo con esta tendencia general, la densidad lineal de la macrofauna total fue significativamente mayor en los valles. En cambio, la riqueza de especies por transecto no fue diferente entre valles y cúspides. Las especies de la macrofauna presentaron claros patrones de zonación, evidenciados por marcadas diferencias de densidad entre los niveles alto, medio y bajo del intermareal. El patrón de zonación de la macrofauna total, así como los patrones de las especies, Excirolana hirsuticauda, Orchestoidea tuberculata y Euzonus heterocirrus, se diferenciaron entre valles y cúspides. Se concluye que la variabilidad espacial de la densidad y zonación de la macrofauna a lo largo de la playa analizada, estaría asociada en algunas especies a variaciones en la morfodinámica intermareal.
\end{abstract}

Palabras claves: macrofauna, intermareal, morfodinámica, parchosidad, playa de arena, zonación.

\section{Macrofaunal spatial distribution on a exposed sandy beach of central Chile: effects of intertidal morphodynamic}

\begin{abstract}
Although spatial heterogeneity (patchiness) is common in sandy beaches, it has been poorly studied. Patchiness in sandy beach populations has been well documented, but its explanation is usually unknown. In the present paper we propose that the spatial variability of intertidal morphodynamic affect the macrofauna spatial distribution (zonation) and abundance along the beach. We analyzed in a sandy beach of central Chile the effects of the spatial variability of the intertidal morphodynamic, upon macrofaunal density and zonation, by comparing sites of contrasting morphology: bays and horns. In comparison, bays were zones of gentler slope, greater intertidal amplitude, lesser swash frequency, and have lesser percentage of coarse sand than horns. In general, the macrofaunal species tend to be more abundant on bays, but only Excirolana braziliensis showed significantly differences. Accordingly with this general trend, the total macrofauna density was significantly higher on bays. By contrast, the species richness per transect was not different between bays and horns. The species of the macrofauna showed evident zonation patterns, which were demonstrated by important differences of abundance among intertidal levels (i.e., high, mid, low). The zonation of the macrofauna, as well as the zonation patterns of Excirolana hirsuticauda, Orchestoidea tuberculata and Euzonus heterocirrus differed between bays and horns. We concluded that the spatial variability of abundance and zonation alongshore, would be associated in some species with variations in the intertidal morphodynamic.
\end{abstract}

Key words: macrofauna, intertidal, morphodynamic, patchiness, sandy beach, zonation. 


\section{INTRODUCCIÓN}

La heterogeneidad espacial (parchosidad) es un fenómeno muy común en la naturaleza (e.g., Watt, 1947; Roughgarden, 1977; Kotliar y Wiens, 1990; Levin, 1992). De hecho, la mayor parte de las especies se distribuyen en el espacio según un patrón agregado (e.g., Hulbert, 1990; Levin, 1992). Las agregaciones espaciales de organismos, o parches, pueden tener diferentes causas, que van desde fenómenos conductuales (e.g., Gendron, 1977), reclutamiento (e.g., Navarrete y Castilla, 1990; Raimondi, 1990), competencia (e.g., Fleeger y Gee, 1986), disponibilidad de alimento (e.g., Palmer et al., 1996), perturbación (e.g., Thistle, 1981; Pickett y White, 1985; Sousa, 1985) y factores físicos (e.g., Jakson y Caldwell, 1993; Chapman y Underwood, 1994).

La fauna de invertebrados mayores de $1 \mathrm{~mm}$ (i.e., macrofauna) que habita la zona intermareal de playas de arena no constituyen la excepción (e.g., McLachlan, 1983, 1989). La densidad de la macrofauna intermareal sufre importantes variaciones espaciales a lo largo de las playas de arena. A pesar de que esta heterogeneidad espacial es un fenómeno recurrente en estos sistemas (McLachlan, 1983, 1989) ha recibido escasa atención por parte de los ecólogos de playas. Si bien algunos estudios recientes han descrito claramente la ocurrencia de parchosidad a diferentes escalas espaciales en poblaciones de invertebrados de playas de arena (e.g., James y Fairweather, 1996), en general se desconocen los factores causales de la misma.

La morfodinámica de la zona intermareal constituye dentro del contexto de la «ecología de playas de arena», el factor clave explicativo de la distribución y densidad de la macrofauna intermareal (e.g., McLachlan 1989; McLachlan et al., 1993, Jaramillo et al., 1993). La morfodinámica está asociada a una serie de variables físicas altamente correlacionadas: tipo de sedimento, pendiente de la playa, tipo de oleaje y clima de marea. De acuerdo a su morfodinámica, las playas de arena se pueden clasificar en tres tipos: 1) disipativas: playas de arena fina, suave pendiente, olas de gran energía que se disipa en una amplia zona intermareal y baja frecuencia de barrido de la ola en el intermareal (frecuencia de barrido, o del «swash») y de traspaso de la línea de efluente (línea que marca el límite entre la zona saturada e insaturada de agua) (Short y Wrigth, 1983; McArdle y McLachlan 1991; 1992); 2) reflectivas: playas de arena gruesa, alta pendiente, olas de baja energía que golpean en una reducida zona intermareal donde se refleja, alta frecuencia del barrido y de traspasos de la línea de efluente (Short y Wrigth; McArdle y McLachlan 1991; 1992); y 3) intermedias: playas de características intermedias entre disipativas y reflectivas (Short y Wrigth, 1983; McArdle y McLachlan 1991; 1992).

El efecto de la variabilidad de la morfodinámica intermareal sobre atributos poblacionales y comunitarios ha sido ampliamente estudiado a escala regional (i.e., entre playas) (e.g., McLachlan, 1990; Defeo et al., 1992; Jaramillo et al., 1993; Jaramillo y McLachlan, 1993; McLachlan et al., 1993, Borzone et al., 1996; McLachlan et al., 1996); sin embargo existen muy pocos estudios a escala local (i.e., intra playa) (sin embargo ver McLachlan y Hesp, 1984).

A nivel local, McLachlan y Hesp (1984) observaron en una playa reflectiva de Australia importantes variaciones espaciales en la morfodinámica intermareal, asociadas principalmente a dos tipos de morfologías contrastantes producidas por el patrón de circulación en la zona de barrido de la ola, valles y cúspides. En los valles el sedimento es comparativamente más fino y la pendiente más suave, lo que determina que la frecuencia de barrido sea más baja que en las cúspides. En asociación a estas morfologías contrastantes, McLachlan y Hesp (1984) encontraron importantes diferencias en la densidad y distribución de varias especies de invertebrados.

En playas intermedias también existen variaciones espaciales en la morfodinámica del intermareal (i.e., valles y cúspides) producidas por el patrón de circulación costera tipo «rip current» (Short y Wrigth, 1983; Short, 1996), aunque menos acentuadas que en playas reflectivas. En este trabajo proponemos que la variabilidad espacial de la morfodinámica intermareal en playas intermedias, afecta a la densidad y distribución espacial (zonación) de la macrofauna a lo largo de la playa. En tal sentido, analizamos las respuestas de la macrofauna de la playa San Carlos (ca. 333' S, $\left.71^{\circ} 36^{\prime} \mathrm{W}\right)$ (Chile central) a las variaciones de la morfodinámica intermareal, en base a la comparación entre valles y cúspides.

\section{MATERIALES Y MÉTODOS}

Este estudio fue realizado en Chile central, específicamente en la playa San Carlos (ca. 3330'S, 71'36'W), localizada aproximadamente a $1 \mathrm{~km}$ de 
Las Cruces (Fig. 1). San Carlos es una playa expuesta de unos $5 \mathrm{~km}$ de longitud, de características morfodinámicas tipo playa intermedia (sensu, Short y Wrigth, 1983), pendiente suave en la playa subaérea (i.e., encima de la línea de resaca) $(2.5 \pm$ $1.1 \%)$ y relativamente pronunciada en la zona intermareal $(5.4 \pm 1.2 \%)$, arena fina $(0.108 \pm 0.015$ $\mathrm{mm})$ y una amplitud intermareal intermedia (35 $40 \mathrm{~m}$ ). La topografía a lo largo de la playa San Carlos presenta pequeñas ondulaciones, producidas por la ocurrencia de valles y cúspides espaciadas cada 30-40 m.

En noviembre de 1996 se muestrearon durante la marea baja 6 transectos, 3 ubicados en zonas de valles y 3 en zonas de cúspides (Fig. 2). Cada

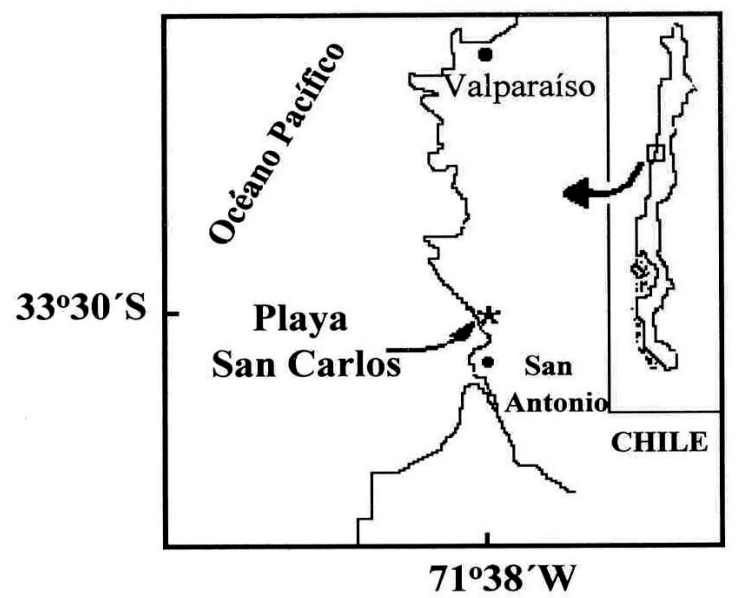

transecto fue muestreado según un diseño estratificado. Se definieron 3 estratos: 1) intermareal alto: 30m encima de la línea de resaca, 2) intermareal medio: zona comprendida entre las líneas de resaca y de efluente (12 - $15 \mathrm{~m})$ e 3 ) intermareal bajo: zona comprendida entre la línea de efluente y el límite inferior de marea (20 m) (ver Fig. 2). En cada estrato se recolectaron 2 muestras con un cubo metálico de $0.1 \mathrm{~m}^{2}$ de superficie y $30 \mathrm{~cm}$ de profundidad. Las muestras fueron filtradas en cernidores de $1 \mathrm{~mm}$ de malla, y los organismos retenidos fueron fijados con formalina al $10 \%$ para ser posteriormente identificados y cuantificados.

Se construyeron los perfiles y estimaron las pendientes de cada transecto según la metodología propuesta por Emery (1961). Se recolectó una muestra de sedimento por estrato, que posteriormente fue analizada mediante tamizado en columna de tamices con 1 phi de intervalo.

La densidad lineal (ind. ${ }^{-1}$, estimada por interpolación lineal) de cada especie y de toda la macrofauna, y la riqueza de especies por transecto se comparó mediante una prueba de Student pareada. La variabilidad espacial de la densidad (ind. $\mathrm{m}^{-2}$ ) de cada especie en relación a la posición en el intermareal, fue analizada mediante análisis de varianza (ANDEVA) de una vía de factor fijo (factor: estrato; niveles: intermareal alto, medio y bajo). Los patrones de zonación de cada especie fueron comparados entre valles y cúspides mediante pruebas de Chi cuadrado.

Figura 1. Area de estudio.

Figure 1. Study area.

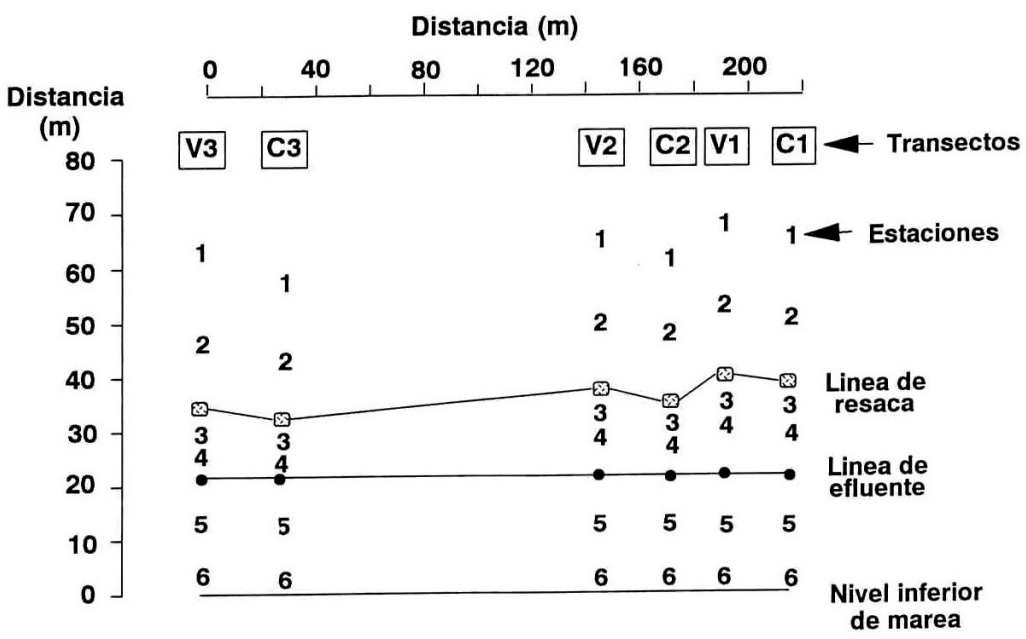

Figura 2. Diseño de muestreo. Ubicación de los transectos y estaciones de muestreo. $V_{i}=$ transectos en valles, $C_{i}=$ transectos en cúspides. Se señala la ubicación de las líneas de resaca y de efluente, $y$ el nivel inferior de marea.

Figure 2. Sampling design. Location of transects and sampling stations. $\mathrm{V}_{\mathrm{i}}=$ bay transects, $C_{i}=$ horn transects. The drift and efluent lines, and the low level tide are indicated. 


\section{RESULTADOS}

Comparativamente los valles fueron sectores de menor pendiente, mayor amplitud intermareal (i.e., zona comprendida entre el nivel de marea baja y la línea de resaca) (ver Fig. 3) y de menor frecuencia de barrido que las cúspides (valle: $2.58 \pm 0.29 \mathrm{~min}^{-}$ ${ }^{1}$, cúspide: $3.25 \pm 0.36 \mathrm{~min}^{-1}$; prueba t pareada de una cola, $\mathrm{t}=2.86, \mathrm{p}=0.03$ ). La granulometría de valles y cúspides no se diferenció significativamente en términos de grano medio, desviación estandar, asimetría y curtosis, sin embargo, las cúspides presentaron un mayor porcentaje de arena gruesa que los valles (valle: $5.42 \pm 3.94 \%$, cúspides: $3.86 \pm$ $3.27 \%$, prueba de $\mathrm{t}$ pareada, $\mathrm{t}=3.057, \mathrm{p}=0.028$ ).

La macrofauna de la playa San Carlos, tanto en valles como cúspides, estuvo integrada por 8 especies, 2 isópodos cirolánidos (Excirolana braziliensis y E. hirsuticauda), 1 anfípodo talítrido (Orchestoidea tuberculata), 1 decápodo anomuro (Emerita analoga), 2 poliquetos (Scolelepis chilensis y Euzonus heterocirrus) y 2 insectos coleópteros (Phalerisidia maculata y Eriopis conexa). Los crustáceos dominaron la comunidad en términos de densidad, constituyendo un $93.9 \%$ del total, mientras que poliquetos $(5.4 \%)$ e insectos $(0.7 \%)$ representaron una fracción muy baja de la densidad total.

En general las especies de la macrofauna, principalmente los crustáceos, tendieron a presentar las mayores densidades lineales en los valles, pero sólo para Excirolana braziliensis esas diferencias fueron significativas (Fig. 4a). De acuerdo con esta tendencia general, la densidad lineal de la macrofauna to- tal fue significativamente mayor en los valles (Fig. 4b). En cambio, la riqueza de especies por transecto no fue diferente entre valles y cúspides (Fig. 4c).

A excepción de Excirolana hirsuticauda, Scolelepis chilensis y Eriopis conexa que fueron excluidas del análisis por presentar muy bajas densidades, todas las especies de la macrofauna presentaron densidades significativamente diferentes $(p<0.05)$ entre los niveles alto, medio y bajo del intermareal, lo que constituye una clara evidencia de zonación. Phalerisidia maculata y Eriopis connexa ocurrieron en el intermareal alto, extendiendo esta última su distribución hasta el intermareal medio (ver Fig. 5). Orchestoidea tuberculata presentó las mayores densidades alrededor de la línea de resaca, dominando conjuntamente con Excirolana braziliensis y E. hirsuticauda el intermareal medio (ver Fig. 5). Scolelepis chilensis ocupó el intermareal medio y bajo, mientras que Emerita analoga y Euzonus heterocirrus dominaron la zona baja del intermareal (ver Fig. 5).

El patrón de zonación de la macrofauna total, así como los patrones de las especies, Excirolana hirsuticauda, Orchestoidea tuberculata y Euzonus heterocirrus, se diferenciaron significativamente (prueba de Chi cuadrado, $\mathrm{p}<0.01$ ) entre valles y cúspides (Fig. 5). Los patrones espaciales de Excirolana hirsuticauda y Euzonus heterocirrus tuvieron mayor sesgo hacia el intermareal bajo en los valles, mientras que el patrón de Orchestoidea tuberculata se hizo más leptocurtico alrededor de la línea de resaca en los valles (ver Fig. 5).
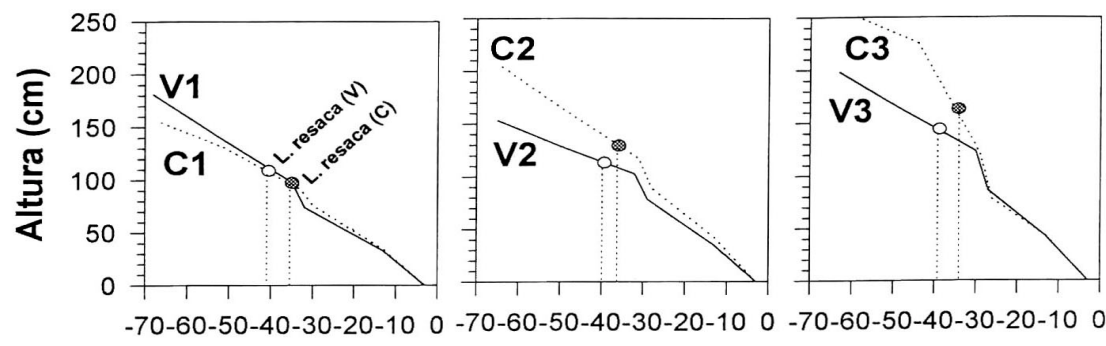

Distancia al nivel inferior de marea $(m)$

Figura 3. Perfiles de los transectos analizados. $V_{i}=$ transectos en valles, $C_{i}=$ transectos en cúspides. Se señala la ubicación de la línea de resaca en cada transecto.

Figure 3. Transect profiles. $V_{i}=$ bay transects, $C_{i}=$ horn transects. The location of drift line are indicated on each transect. 


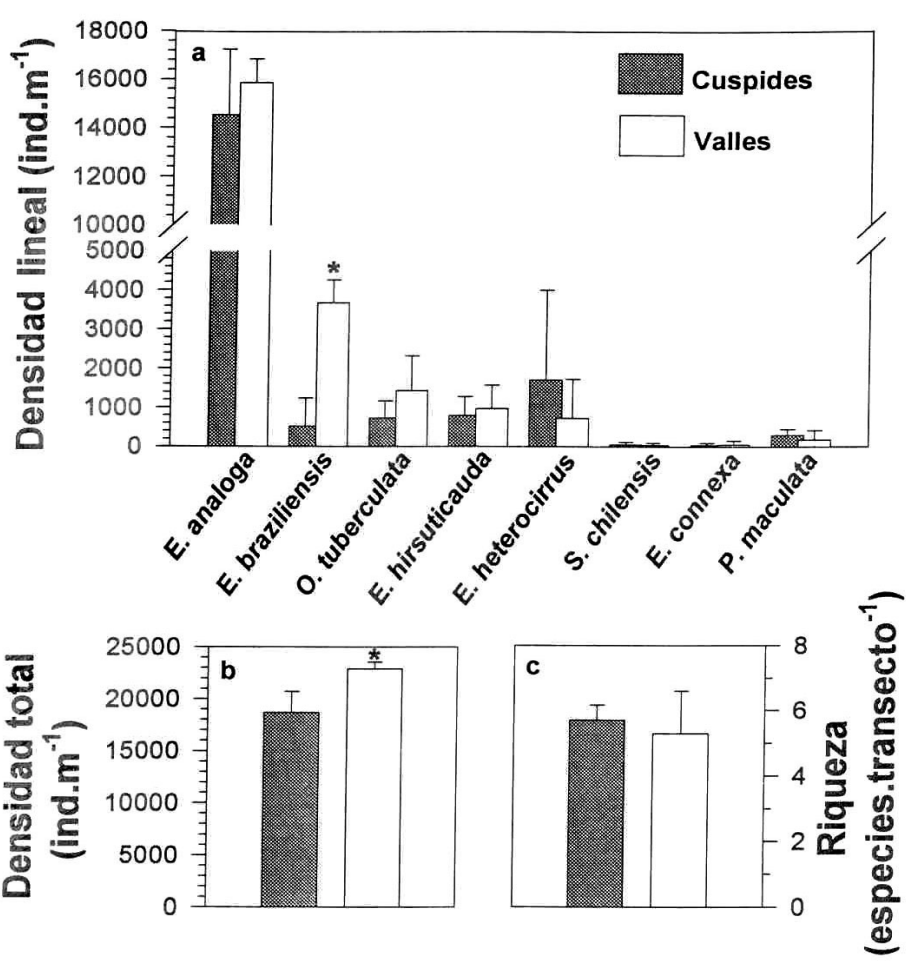

Figura 4. Valores medios \pm 1 desviación standard de: a) densidad lineal por especie discriminada por tipo de transecto (i.e., valle o cúspide), b) densidad lineal total de la macrofauna discriminada por tipo de transecto, y c) riqueza de especies por transecto. El asterisco (*) significa que la media indicada es significativamente mayor que la media correspondiente al otro tipo de transecto (Prueba de $t$ pareada, $\mathbf{p}<0.05$ ).

Figure 4. Mean values \pm 1 standard deviation of: a) species lineal density per transect type (i.e., bays or horns), b) total lineal density of the macrofauna per transect type, and c) species richness per transect. The asterisk (*) indicate that the mean value marked is significantly higher than the mean value of the other transect type (Student test for dependent observations, $\mathbf{p}<0.05$ ).

\section{PATRONES DE ZONACION}

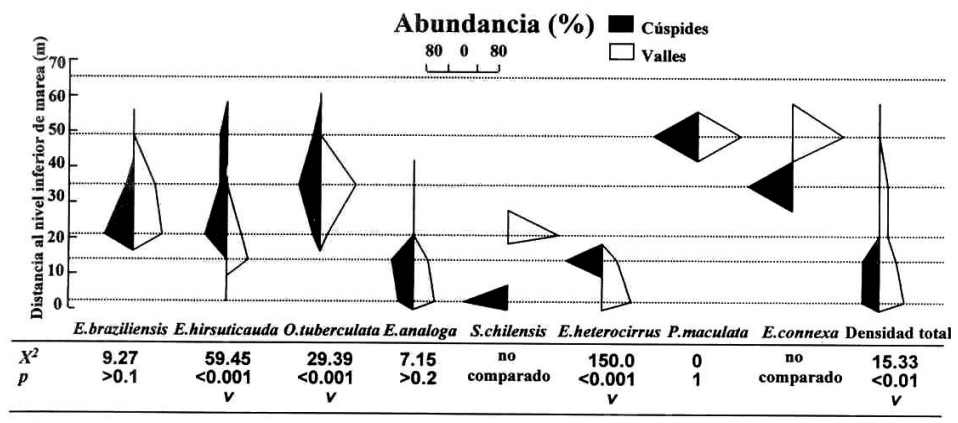

Figura 5. Patrones de zonación de la macrofauna discriminados por tipo de transecto (i.e., valle o cúspide). Se resumen en la parte inferior los resultados de las comparaciones estadísticas (Prueba de Chi cuadrado).

Figure 5. Zonation patterns of the macrofauna discriminated by transect type (i.e., bays or horns). In the low part of the figure are summarized the results of the statistical comparisons (Chi- square Test). 


\section{DISCUSIÓN}

Se detectaron variaciones morfodinámicas a lo largo de la playa San Carlos asociadas a la ocurrencia de valles y cúspides, siendo los valles sectores con menor pendiente, mayor amplitud intermareal, menor frecuencia de barrido y con un menor porcentaje de arena gruesa que las cúspides. La caracterización física de los sectores tipo valle y cúspide en la playa intermedia de San Carlos coincidió con lo descrito por McLachlan \& Hesp (1984) para una playa reflectiva de Australia, aunque las diferencias encontradas entre valles y cúspides en el presente trabajo no fueron tan amplias como en el caso australiano. Esto indica que el patrón de circulación costera en playas de tipo morfodinámico intermedio es, al igual que en playas reflectivas, capaz de generar diferencias locales significativas en la morfodinámica intermareal.

En relación a estas diferencias en la morfodinámica intermareal asociadas a la presencia de valles y cúspides, se observaron en la playa San Carlos variaciones tanto en la densidad como en la zonación de algunas especies de la macrofauna. La macrofauna (total) ocurrió en mayores densidades en los valles, debido a que los crustáceos, el grupo dominante, tendieron a ser más abundantes en estos sectores. Sin embargo, a nivel de especie, solamente en el caso de Excirolana braziliensis la diferencia fue estadísticamente significativa. Esta tendencia de la macrofauna a concentrarse en los valles corrobora las observaciones de McLachlan \& Hesp (1984), quienes encontraron las mayores densidades de bivalvos filtradores asociadas a estos sectores. La mayor concentración de individuos en los valles podría explicarse por dos vías no excluyentes: 1) transporte pasivo de individuos por el agua hacia los valles, o 2) selección de hábitat. En el primer caso, los individuos podrían ser arrastrados desde las cúspides hacia los valles, por la corriente que se desplaza en esa dirección cuando avanza el tren de olas por el intermareal. En el caso de la selección activa de hábitat, los individuos podrían ser favorecidos en los valles por varios factores. El clima de marea en los valles, que se asemeja más a la condición disipativa (i.e., barridos más largos y menos frecuentes), sería más «benigno» para la macrofauna por varios motivos: 1) disminución de la probabilidad de ser removidos del intermareal por la acción del oleaje, 2) mayor tiempo de alimentación, y 3) mayor disponibilidad de alimento (i.e., mayor acumulación de restos orgánicos en la línea de resaca). Por otra parte, en los valles las condiciones «favorables» de humectación (i.e., aquellas que permiten el movimiento y enterramiento de los individuos; Brown, 1983), se extienden hacia el intermareal alto algunos metros más que en las cúspides, por lo cual la zona habitable se amplía en los valles.

La habilidad de los organismos para seleccionar activamente un hábitat determinado requiere de la capacidad de exploración. En el caso de la macrofauna de playas de arena, especialmente en los crustáceos, las especies son móviles por lo cual la capacidad de exploración está presente. Por ejemplo, Orchestoidea tuberculata se moviliza activamente por el intermareal durante marea baja, y principalmente durante la noche cuando presenta el máximo de actividad (Jaramillo et al., 1980). Asimismo, Excirolana hirsuticauda, E. braziliensis (e.g., Jaramillo \& Fuentealba, 1993) y Emerita analoga (obs. pers.) también realizan importantes desplazamientos en el intermareal por la interfase agua-sedimento durante la marea alta.

Si bien las características morfodinámicas de valles y cúspides correspondieron en términos relativos a las condiciones de playas disipativas y reflectivas respectivamente, la riqueza de especies no varió de acuerdo a lo esperado. Ha sido documentado que la riqueza de especies en playas de arena decrece significativamente a medida que las condiciones se hacen más reflectivas (e.g., McLachlan, 1990; Defeo et al., 1992; Jaramillo et al., 1993; Jaramillo y McLachlan, 1993; McLachlan et al., 1993, Borzone et al., 1996; McLachlan et al., 1996), sin embargo, la riqueza y composición de especies fue igual en valles y cúspides. Esto indicaría que a pesar de que las diferencias morfodinámicas entre valles y cúspides pueden alterar significativamente la densidad de algunas especies, estas diferencias no son lo suficientemente intensa como para determinar la exclusión local de especies. Aunque también podría ocurrir que valles y cúspides se comporten como un sistema fuente-sumidero (Pulliam, 1988), es decir, la continua inmigración de individuos desde los valles podría ser el factor responsable de la persistencia de las poblaciones de las cúspides.

Las variaciones morfodinámicas entre valles y cúspides también tuvieron efectos sobre los patrones de zonación de algunas especies. Excirolana hirsuticauda y Euzonus heterocirrus aumentaron sus densidades relativas hacia el intermareal bajo en los 
valles, lo que podría ser explicado por las condiciones «benignas» del clima de marea en estos sectores, en comparación a las cúspides. Por su parte, Orchestoidea tuberculata presentó un patrón de distribución que se hizo más leptocurtico en los valles. En estos sectores, la densidad relativa de Orchestoidea tuberculata alrededor de la línea de resaca fue mayor en los valles, lo que podría ser explicado por la mayor concentración de alimento que existiría alrededor de esta línea en los valles.

En resumen, tanto la densidad como la zonación de algunas especies de la macrofauna de la playa San Carlos, se vio afectada por la variabilidad espacial de la morfodinámica intermareal asociada a la ocurrencia de valles y cúspides. Si bien las diferencias entre valles y cúspides en playas intermedias, como San Carlos, no son tan marcadas como en playas reflectivas (e.g., McLachlan y Hesp, 1984), parecen ser suficientes como para afectar a algunas especies de la macrofauna en términos de su distribución y densidad. En tal sentido, se concluye que la heterogeneidad espacial de la macrofauna intermareal de playas intermedias podría explicarse en parte por la variabilidad de la morfodinámica intermareal asociada a la presencia de valles y cúspides.

\section{AGRADECIMIENTOS}

Los autores agradecen la colaboración en terreno de los alumnos del curso Bio-321B «Zoología de invertebrados marinos» de la Pontificia Universidad Católica de Chile, versión 1996. Asimismo se agradecen los comentarios y observaciones realizadas por tres revisores anónimos. José Miguel Fariña es becario CONICYT y Alejandro Brazeiro es becario de la Pontificia Universidad Católica de Chile.

\section{REFERENCIAS}

Borzone, CA.; J.R.B. Souza y A.G. Soares. 1996. Morphodynamics influence on the structure of inter and subtidal macrofaunal communities of subtropical sandy beaches. Rev. Chi. Hist. Nat., 69: 565-577.

Brown, A.C. 1993. The ecophysiology of sandy beach animals: a partial review. Sandy beaches as ecosystem. McLachlan, A. y T. Erasmus, W. Junks Publishers, The Hague, 575-605.
Chapman, M.G. y A.J. Underwood. 1994. Dispersal of the intertidal snail, Nodilittorina pyramidalis, in response to the topography complexity of the substratum. J. Exp. Mar. Biol. Ecol., 179: 145-169.

Defeo, O; E. Jaramillo y A. Lyonnet. 1992: Community structure and intertidal zonation of the macroinfauna on the atlantic coast of Uruguay. J. Coast. Res., 8(4): 830-839.

Emery, K.O. 1961. A simple method of measuring beach profiles. Limnol. Oceanogr., 6: 90-93.

Fleeger, J.W. y J.M. Gee. 1986. Does interference competition determine the vertical distribution of meiobenthic copepods? J. Exp. Mar. Biol. Ecol., 95: 173-181.

Gendron, R.P. 1977. Habitat selection and migratoty behaviour of the intertidal gastropod Littorina littorea (L.). J. Animal Ecol., 46: 79-92.

Hulbert, S.H. 1990. Spatial distribution of the montane unicorn. Oikos, 58: 257-271.

Jakson, R.B. y M.M. Caldwell. 1993. Geostatistical patterns of soil heterogeneity around individal perennial plants. J. Ecol., 81: 683-693.

James, R.J. y P.G. Fairweather. 1996. Spatial variation of intertidal macrofauna on sandy ocean beach in Australia. Estuar. Coast. Shelf Sci., 43: 81-107.

Jaramillo, E.; W. Stotz; C. Beltrán; J. Navarro; C. Roman y C. Varela. 1980. Actividad locomotriz de Orchestoidea tuberculata (Amphipoda, Talitridae) sobre la superficie de una playa arenosa del Sur de Chile (Mehuin, Provincia de Valdivia). Stud. Neotrop. Faun. Envir., 15: 9-33.

Jaramillo, E., y S. Fuentealba. 1993. Down-shore zonation of two cirolanid isopods during two spring - neap tidal cycles in a sandy beach of south central Chile. Rev. Chil. Hist. Nat., 66: 439-454.

Jaramillo, E., A. McLachlan y P. Coetzee. 1993. Intertidal zonation patterns of macroinfauna over a range of exposed sandy beaches in south-central Chile. Mar. Ecol. Prog. Ser., 101: 105-118.

Jaramillo, E. y A. McLachlan. 1993. Community and population response of the macroinfauna to physical factors over a range of exposed sandy beaches in south-central Chile. Estuar. Coast. Shelf Sci., 37: 615-624. 
Kotliar, N.B. y J.A. Wiens. 1990. Multiple scale of pathiness and patch structure: a hierarchical framework for the study of heterogeneity. Oikos, 59: 253-260.

Levin, S.A. 1992. The problem of pattern and scale in ecology. Ecology, 73(6): 1943-1967.

McArdle, S.B. y A. McLachlan. 1991. Dynamics of the swash zone and effluent line on sandy beaches. Mar. Ecol. Prog. Ser., 76: 91-99.

McArdle, S.B. y A. McLachlan. 1992. Sand beach ecolgy: swash features relevant to the macrofauna. J. Coast. Res., 8: 398-407.

McLachlan, A. 1983. Sandy beach ecology: a review. Sandy beach as ecosystem. McLachlan A. y T. Erasmus, Junk. The Hague, 321-380.

McLachlan, A. 1988. Behaviour adaptation of sandy beach organims: an ecological perspective. Beheviour adaptation to intertidal life. Chelazzi, G. y M. Vannini, Plenum Publishing Corporation, 449-475.

McLachlan, A. 1990. Dissipative beaches and macrofauna communities on exposed intertidal sands. J. Coast. Res., 6: 57-71.

McLachlan, A.; A.D. Ruyck y N. Hacking. 1996. Community structure on sandy beaches: patterns of richness and zonation in relation to tide range and latitud. Rev. Chi. Hist. Nat., 69: 451-467.

McLachlan, A. y P. Hesp. 1984. Faunal response to morphology and water circulation of sandy with cusps. Mar. Ecol. Prog. Ser., 19: 133-144.

McLachlan, A.; E. Jaramillo; T.E. Donn y F. Wessels. 1993. Sandy beach macrofauna communities and their control by physical environment: a geographical comparision. J. Coast. Res., 15: 27-38.

Navarrete, S.A.y J.C. Castilla. 1990. Barnacle walls as mediators of intertidal mussel recruitment: effects of patch size on the utilization of space. Mar. Ecol. Prog. Ser., 68: 113-119.
Palmer, M.A.; P. Arensburger; A.P. Martin y D.W. Denman. 1996. Disturbance and patch-specific response: the interactive effects of woody debris and floods on lotic invertebrates. Oecologia, 105: 247-257.

Pickettt, S.T.A. y P.S. White. 1985. Patch dynamics: a synthesis. The ecology of natural disturbance and patch dynamics. Pickettt, S.T.A. y P.S. White, Academic Press Inc, New York, 371-385.

Pulliam, H.R. 1988. Sources, sinks and population regulation. Am. Nat., 132(5): 652-661.

Raimondi, P.T. 1990. Patterns, mechanisms, consequences of variability in settlement and recruitment of intertidal barnacles. Ecol. Monogr., 60: 283-309.

Roughgarden, J.D. 1977. Patchiness in the spatial distribution of a population caused by stochastic fluctuations in resources. Oikos, 29: 52-59.

Short, A.D. 1966. The role of wave height, period, slope, tide range and embaymentisation in beach classifications: a review. Rev. Chi. Hist. Nat., 69: 589-604.

Short, A.D. y L.D. Wrigth. 1983. Physical variability of sandy beaches. Sandy beaches as ecosystem. McLachlan, A. y T. Erasmus, W. Junks Publishers, The Hague, 133-144.

Sousa, W.P. 1985. Disturbance and patch dynamics on rocky intertidal shores. The ecology of natural disturbance and patch dynamics. Pickettt, S.T.A. y P.S. White, Academic Press Inc, New York, 101124.

Thistle, D. 1981. Natural physical disturbance and communities of marine soft bottoms. Mar. Ecol. Prog. Ser., 6: 223-228.

Watt, A.S. 1947. Pattern and procces in the plant community. J. Ecol., 36: 1-22. 